\title{
Pregnancy and lactation Dietary fat and Breast Cancer Risk in the Female Offspring
}

\author{
Behnoush Hossein-Nia ${ }^{1, *}$, Ali Tarighat-Esfanjani ${ }^{2}$ \\ ${ }^{1}$ Department of Nutrition and Food Sciences, Tabriz University of Medical Sciences, \\ Tabriz, Iran \\ ${ }^{2}$ Department of Nutrition Science, Nutrition Research Center, Faculty of Nutrition, \\ Tabriz University of Medical sciences, Tabriz, Iran
}

${ }^{*}$ Corresponding author: Behnoush Hossein-Nia, Department of Nutrition and Food Sciences, Tabriz University of Medical Sciences, Tabriz, Iran.E-mail: behnoosh015@

DOI: $10.21859 /$ mci-supp-79 gmail.com, Tel: +98-9375882945

\author{
Keywords: \\ Breast Cancer Fat \\ Dietary Fat \\ Pregnancy \\ Lactation \\ Fetal
}

\begin{abstract}
Introduction: Breast cancer is the most common cancer in women and a global public health problem. Evidence shows that breast cancer may originate during early life. Although diet has been implicated as playing a major role in prevention of breast cancer, we do not know what dietary factors are responsible for initiating and developing breast cancer. The objective of this review was to assess a possible role of dietary fat intake in fetal and lactation in increases breast cancer risk in adulthood.

Materials and Methods: This review includes a summary of original article and review in full text from 2000- 2017 and was performed using pubmed, science direct, scopus, and google scholar databases for terms: breast cancer, diet, dietary fat, pregnancy, lactation, fetal, fatty acid, w3, w6.

Results: According to our search 6 article, including 6 animal, 2 review was investigated the relationship between maternal fat intake and breast cancer risk in the female offspring. Intake of canola oil, w3 and fish oil delayed mammary gland tumor development in animal. results showed intake of a lard-based high-fat diet rich in SFA and fat n-6 polyunsaturated acid during pregnancy increases female offspring's mammary cancer risk, while a study suggest an exposure to a lard-based HF diet during early life changes the fatty acid profile and transcriptional network in mammary gland in young adult rats these changes appear to be consistent with reduced mammary cancer risk.

Conclusions: The literature suggests that the breast cancer risk of offspring can be programmed by maternal dietary fat intake, but further researches are recommended.
\end{abstract}

